\title{
Aptidão cardiorrespiratória e fatores de risco para hipertensão arterial em adolescentes
}

\author{
Cardiorespiratory fitness and risk factors for high blood pressure among adolescents \\ Cleilton Holanda Pereira ${ }^{1,2}$, Evanice Avelino de Souza ${ }^{2,4}$, Julia Aparecida Devidé Nogueira ${ }^{1,2}$, Nicolino Trompieri Filho ${ }^{2,3}$ \\ ${ }^{1}$ Programa de Pós-Graduação em Educação Física da Universidade de Brasília (UnB), Brasília, DF. \\ ${ }^{2}$ Grupo de Pesquisa em Promoção da Atividade Física e Saúde na Escola (GPAFSE), Universidade Federal do Ceará (UFC), Fortaleza, CE. \\ ${ }^{3}$ Faculdade de Educação, UFC, Fortaleza, CE. \\ ${ }^{4}$ Faculdade Nordeste (Fanor), DeVry Brasil, Fortaleza, CE.
}

\section{RESUMO}

Objetivos: Analisar possíveis associações do nível de pressão arterial com a aptidão cardiorrespiratória e o índice de massa corporal em adolescentes de nível socioeconômico baixo.

Métodos: O estudo incluiu adolescentes de 14 a 17 anos de uma escola pública em Fortaleza, Ceará, que atende uma população de baixo poder aquisitivo e elevado risco social. Foram aferidos peso, estatura, pressão arterial sistólica e diastólica e aptidão cardiorrespiratória estimada por meio do teste de corrida. A análise estatística incluiu valores percentuais, média, desvio padrão, teste $t$ de Student, Qui-quadrado e regressão logística binária, adotando-se p $<0,05$ como significativo. Foi utilizado o pacote SPSS 17.0 para as análises.

Resultados: Participaram do estudo 308 adolescentes, sendo 177 meninos. Verificou-se que 12,3\% dos adolescentes encontravam-se acima do peso; 75,3\% apresentavam baixa aptidão cardiorrespiratória; e 51\% apresentavam valores elevados de pressão arterial. Os adolescentes cujo índice de massa corporal estava acima dos critérios de saúde apresentaram maior risco de níveis aumentados de pressão arterial sistólica (razão de chances 3,02; intervalo de confiança 95\% 2,94-6,13; $<<0,01$ ) e diastólica (razão de chances 3,41; intervalo de confiança 95\% 1,55-7,49; $\mathrm{p}=0,02)$. A aptidão cardiorrespiratória não apresentou associação estatisticamente significativa com pressão arterial e índice de massa corporal. Conclusões: Em adolescentes de nível socioeconômico baixo, elevados índices de massa corporal foram associados a aumento da pressão arterial. $\mathrm{O}$ excesso de peso, a baixa aptidão cardiorrespiratória e a pressão arterial acima do normal foram prevalentes nessa população.

DESCRITORES: PRESSÃO ARTERIAL; ÍNDICE DE MASSA CORPORAL; APTIDÃO FÍSICA; FATORES DE RISCO; ADOLESCÊNCIA.

\section{ABSTRACT}

Aims: To analyze possible associations of blood pressure level with cardiorespiratory fitness and body mass index in adolescents of low socioeconomic status.

Methods: The study included adolescents aged 14 to 17 years at a public school in Fortaleza, Ceará, which serves a population of low income and high social risk. Weight, height, systolic and diastolic blood pressure were measured, and cardiorespiratory fitness was estimated by the running test. The statistical analysis included percentages, mean, standard deviation, Student $t$ test, chi-square and binary logistic regression, adopting $\mathrm{p}<0,05$ as significant. SPSS 17.0 package was used for analysis.

Results: The study included 308 adolescents, being 177 boys. It was found that $12.3 \%$ of adolescents were overweight; $75.3 \%$ had low cardiorespiratory fitness; and 51\% had high blood pressure. Adolescents whose body mass index was above the health criteria had higher risk of increased levels of systolic blood pressure (odds ratio 3.02; 95\% confidence interval 2.94-6.13; $<<0.01$ ) and diastolic (odds ratio 3.41; $95 \%$ confidence interval $1.55-7.49 ; \mathrm{p}=0.02$ ). Cardiorespiratory fitness had no statistically significant association with blood pressure and body mass index.

Conclusions: In adolescents of low socioeconomic status, high body mass index were associated with increased blood pressure. Excess weight, low cardiorespiratory fitness and blood pressure above normal were prevalent in this population.

KEY WORDS: BLOOD PRESSURE; BODY MASS INDEX; PHYSICAL FITNESS; RISK FACTORS; ADOLESCENCE. 


\section{INTRODUÇÃO}

Atualmente as doenças crônicas não transmissíveis (DCNT) são as principais causas de morbimortalidade no Brasil e no mundo, acarretando elevados custos pessoais, sociais e com os cuidados de saúde. A alta prevalência de DCNT decorre do estilo de vida contemporâneo sedentário associado a uma dieta rica em açúcar, gorduras saturadas e sal. O estabelecimento dos padrões comportamentais que aumentam ou reduzem os riscos de desenvolvimento de DCNT podem ter relação com o nível socioeconômico e educacional. As DCNT se desenvolvem ao longo da vida de forma lenta e gradual, tendo a infância como ponto de partida. Entretanto, a incidência de DCNT vem aumentando em crianças e jovens e ocorrendo em idades cada vez mais precoces. Dentre as DCNT, as doenças cardiovasculares (DCV) são as que mais causam mortes, sendo responsáveis por $48 \%$ da mortalidade mundial. Entre os fatores de risco associados ao desenvolvimento das $\mathrm{DCV}$, está o excesso de gordura corporal (sobrepeso e obesidade), a hipertensão arterial sistêmica (HAS) e as dislipidemias. ${ }^{1-8}$

No Brasil, a exemplo das estatísticas mundiais, o aumento da incidência e prevalência dos fatores de risco para DCV em crianças e adolescentes vem assumindo um caráter epidêmico. ${ }^{9}$ Dados do Ministério da Saúde mostram que $47,0 \%$ dos escolares acima de 12 anos estão com sobrepeso. ${ }^{10}$ Outro estudo de âmbito nacional mostrou que a prevalência de excesso de peso em adolescentes oscilou, nos dois sexos, de $16 \%$ a $19 \%$ nas Regiões Norte e Nordeste e de $20 \%$ a $27 \%$ nas Regiões Sudeste, Sul e Centro-Oeste. ${ }^{11}$ Já para a HAS, são reportadas elevadas prevalências na infância e na adolescência em diversas regiões do Brasil, oscilando entre $2,5 \%$ e $44,7 \%$. $^{8,12-14}$

Por outro lado, um fator de proteção ao desenvolvimento de DCV é a aptidão cardiorrespiratória (ApC), que pode ser definida como a capacidade de realizar e manter atividades físicas durante um período prolongado de tempo, podendo ser avaliada através dos testes de corridas e/ou caminhadas. Atualmente, observa-se uma redução da $\mathrm{ApC}$ entre crianças e adolescentes de vários países do mundo, ${ }^{15,16}$ incluindo o Brasil. ${ }^{3,17}$

Em vista destas considerações, é importante compreender as relações entre fatores de risco para o desenvolvimento de DCV, como excesso de peso e HAS, e um fator de proteção, como a ApC, em crianças e adolescentes. Como o Brasil é um país com dimensões continentais e ampla diversidade cultural, geográfica e socioeconômica, é importante a realização de estudos em diversas regiões e realidades sociais do país. Neste sentido, o presente estudo tem como objetivo verificar os níveis médios de índice de massa corporal (IMC), pressão arterial (PA) e ApC, a prevalência dos desvios da normalidade destas variáveis e as associações entre as mesmas, em adolescentes de uma escola pública em área de risco social na cidade de Fortaleza, estado do Ceará.

\section{MÉTODOS}

O presente estudo foi desenvolvido a partir de um projeto maior de caráter epidemiológico transversal com escolares do ensino médio da rede Liceu, composta por quatro escolas públicas localizadas nos bairros do Centro, Conjunto Ceará, Messejana e Vila Velha, da cidade de Fortaleza, capital do Ceará. O projeto intitulado "Análise da classificação nutricional, hábitos alimentares, prática de atividade física e comportamento sedentário em escolares do colégio Liceu do Ceará" foi aprovado pelo Comitê de Ética em Pesquisa da Universidade Federal do Ceará sob o número 45/10 e acompanha as normas da resolução 196/96 do Conselho Nacional de Saúde, sobre pesquisa envolvendo seres humanos.

$\mathrm{O}$ estudo pesquisou adolescentes entre 14 e 17 anos do Colégio Liceu do bairro Vila Velha, uma área de baixo poder aquisitivo e elevado risco social. Após a autorização da direção da escola, os 771 alunos matriculados no colégio foram convidados a participar da pesquisa, e aqueles que apresentaram interesse receberam o termo de consentimento livre e esclarecido para assinatura dos responsáveis. Foram excluídos da amostra adolescentes com deficiência física e gestantes.

A coleta de dados foi realizada no dia seguinte ao convite, durante o horário letivo. Os professores liberavam os alunos em grupos de cinco para a realização das medidas. Inicialmente os adolescentes informavam os dados pessoais (nome e idade) e seguiam para avaliação antropométrica do peso e estatura corporal. Para aferir o peso, utilizou-se uma balança portátil digital marca Plenna (São Paulo, SP) com capacidade de $120 \mathrm{~kg}$ e precisão de $0,1 \mathrm{~kg}$. Para medir a estatura foi utilizada uma fita métrica não extensível com precisão de $0,1 \mathrm{~cm}$ fixada à parede, de acordo com protocolo já descrito. $^{18}$

A relação estatura por idade dos adolescentes foi avaliada pelo indicador altura/idade nos seguintes pontos de corte segundo as recomendações da Organização Mundial da Saúde (2007): abaixo do 
percentil 3, baixa estatura para idade; no percentil 3 e acima, estatura adequada para a idade. ${ }^{19}$ Para que fosse possível verificar a distribuição do peso dos escolares estudados, foi realizada a classificação mediante intervalo interquartílico. Desta forma, os indivíduos que apresentavam peso no quarto quartil para a faixa etária, foram classificados como alto peso. O IMC foi calculado $\left(\mathrm{kg} / \mathrm{m}^{2}\right)$ e o estado nutricional dos adolescentes foi definido a partir dos pontos de corte (atende/não atende os critérios de saúde) de acordo com o sexo e a idade. ${ }^{18}$ Os alunos com IMC acima do esperado para a faixa etária foram considerados como não atendendo aos critérios de saúde (Quadro 1).

Após a coleta dos dados antropométricos, os adolescentes ficavam sentados por dois minutos e sua PA era aferida sempre pelo mesmo avaliador, utilizando o mesmo equipamento (Oregon Scientific, Modelo BPW 120) de acordo com os procedimentos e critérios estabelecidos pela Academia Americana de Pediatria - The Fourth Report on the Diagnosis, Evaluation, and Treatment of High Blood Pressure in Children and Adolescents. ${ }^{20}$ Foram realizadas duas medidas, com intervalo de dois minutos entre elas, adotando-se o valor médio das mesmas. $\mathrm{O}$ valor absoluto da pressão arterial foi convertido em percentil e classificado de acordo com as recomendações da mesma referência, ${ }^{20}$ que apresenta valores de pressão em percentis $(90$, 95 e 99), sendo classificados da seguinte maneira: Normal: PA < Percentil 90; Pré-Hipertensão: Percentil 90 até $<$ Percentil 95 e Hipertensão Estágio 1: Percentil 95-99+5mmHg.

Por fim, os adolescentes participaram do teste de corrida/caminhada de nove minutos para avaliação da ApC. ${ }^{18} \mathrm{O}$ teste foi realizado em quadra poliesportiva descoberta previamente delimitada. Foi explicada a execução correta do teste, dando ênfase ao fato de que os participantes deveriam correr o maior tempo possível, evitando piques de velocidade intercalados por longas caminhadas. Os participantes foram informados de que não poderiam parar ao longo do trajeto e que se tratava de um teste de corrida, embora fosse possível caminhar eventualmente quando sentissem cansaço. Durante o teste, foi informada ao aluno a passagem do tempo aos três, seis e oito minutos. Ao final do teste soava um apito e os alunos interrompiam a corrida, permanecendo no lugar onde estavam até ser anotada a distância percorrida por cada aluno. A mesma foi reportada em metros e os resultados foram interpretados a partir dos pontos de corte (atende/não atende aos critérios de saúde) estabelecidos de acordo com o sexo e a idade (Quadro 2). ${ }^{18}$
Quadro 1. Pontos de corte para o índice de massa corporal

\begin{tabular}{|c|c|c|}
\hline Idade & IMC meninos & IMC meninas \\
\hline 14 & 22,2 & 22,0 \\
\hline 15 & 23,0 & 22,4 \\
16 & 24,0 & 24,0 \\
17 & 25,4 & 24,0 \\
\hline
\end{tabular}

IMC: índice de massa corporal $\left(\mathrm{kg} / \mathrm{m}^{2}\right)$.

Quadro 2. Pontos de corte para aptidão cardiorrespiratória estimada pelo teste de corrida/caminhada de 9 minutos

\begin{tabular}{|c|c|c|}
\hline Idade & ApC Meninos & ApC Meninas \\
\hline 14 & $1560 \mathrm{~m}$ & $1220 \mathrm{~m}$ \\
\hline 15 & $1634 \mathrm{~m}$ & $1240 \mathrm{~m}$ \\
\hline 16 & $1660 \mathrm{~m}$ & $1256 \mathrm{~m}$ \\
\hline 17 & $1660 \mathrm{~m}$ & $1256 \mathrm{~m}$ \\
\hline
\end{tabular}

ApC: aptidão cardiorrespiratória estimada pelo teste de corrida/caminhada de 9 minutos (metros).

Para o processamento estatístico dos dados, foi realizado inicialmente o teste de KolmogorovSmirnov. Como as variáveis apresentaram-se normais, a análise estatística utilizou média, desvio padrão e frequência percentual, por sexo e idade. Para análise da significância de proporções de adolescentes que atenderam ao critério de saúde nos testes de ApC, IMC e PA entre os sexos, utilizou-se teste do Quiquadrado $\left(\chi^{2}\right)$. A razão de chances $(\mathrm{RC})$ de atender aos critérios considerados normais para a ApC, IMC e a PA foi calculada através da regressão logística binária, adotando-se o intervalo de confiança de $95 \%$. O nível de significância foi fixado em $\mathrm{p} \leq 0,05$, e as análises foram realizadas através do programa SPSS versão 17.0 .

\section{RESULTADOS}

Ao todo, 361 alunos concordaram em participar e, destes, 20 foram excluídos por estarem fora da faixa etária estabelecida; 16 por não apresentarem o termo de consentimento assinado pelo responsável; e 17 por desistência durante a avaliação. Dessa forma, o estudo incluiu uma amostra de 308 crianças (177 meninos e 131 meninas). A maior proporção de adolescentes tinha 16,15 e 17 anos de idade $(33,1 \%, 28,6 \%$ e $26,3 \%$, respectivamente). A maioria encontrava-se no primeiro quartil do peso, ou seja, com peso normal para idade e acima do percentil 3 para estatura. Houve diferenças significativas entre os sexos no peso, estatura e pressão arterial sistólica (PAS) (Tabela 1). 
Tabela 1. Características gerais da amostra de 361 adolescentes entre 14 e 17 anos de uma escola pública em Fortaleza, Ceará, de acordo com o sexo

\begin{tabular}{|c|c|c|c|c|c|c|c|}
\hline \multirow{2}{*}{ Variáveis } & \multicolumn{2}{|c|}{ Meninos } & \multicolumn{2}{|c|}{ Meninas } & \multicolumn{2}{|c|}{ Total } & \multirow{2}{*}{$\mathbf{p}^{*}$} \\
\hline & $\mathbf{n}$ & $(\%)$ & n & $(\%)$ & n & (\%) & \\
\hline \multicolumn{8}{|l|}{ Idade (anos) } \\
\hline 14 & 20 & $(11,3)$ & 17 & $(13,0)$ & 37 & $(12,0)$ & \multirow{4}{*}{0,92} \\
\hline 15 & 52 & $(29,4)$ & 36 & $(27,5)$ & 88 & $(28,6$ & \\
\hline 16 & 57 & $(32,2)$ & 45 & $(34,4)$ & 102 & $(33,1)$ & \\
\hline 17 & 48 & $(27,1)$ & 33 & $(25,2)$ & 81 & $(26,3)$ & \\
\hline \multicolumn{8}{|l|}{ Peso (kg) } \\
\hline $1^{\circ}$ Quartil & 64 & $(36,2)$ & 17 & (13) & 81 & $(26,3)$ & \multirow{4}{*}{0,01} \\
\hline $2^{\circ}$ Quartil & 52 & $(29,4)$ & 25 & $(19,1)$ & 77 & (25) & \\
\hline $3^{\circ}$ Quartil & 34 & $(19,2)$ & 42 & $(32,1)$ & 76 & $(24,7)$ & \\
\hline $4^{\circ}$ Quartil & 27 & $(15,3)$ & 47 & $(35,9)$ & 74 & (24) & \\
\hline \multicolumn{8}{|l|}{ Estatura $(\mathrm{cm})$} \\
\hline$<$ percentil 3 & 15 & $(8,5)$ & 28 & $(21,4)$ & 43 & (14) & \multirow{2}{*}{0,01} \\
\hline$\geq$ percentil 3 & 162 & $(91,5)$ & 103 & $(78,6)$ & 265 & (86) & \\
\hline \multicolumn{8}{|c|}{ Índice de massa corporal (kg/m²) } \\
\hline Baixo peso & 1 & $(0,6)$ & 3 & $(2,3)$ & 4 & $(1,3)$ & \multirow{4}{*}{0,06} \\
\hline Adequado & 146 & $(82,5)$ & 118 & $(90,1)$ & 264 & $(85,7)$ & \\
\hline Sobrepeso & 24 & $(13,6)$ & 8 & $(6,1)$ & 32 & $(10,4)$ & \\
\hline Obesidade & 6 & $(1,9)$ & 2 & $(0,6)$ & 8 & $(2,6)$ & \\
\hline \multicolumn{8}{|c|}{ Pressão arterial sistólica (mm/Hg) } \\
\hline$<$ percentil 90 & 77 & $(43,5)$ & 74 & $(56,5)$ & 151 & $(49,0)$ & \multirow{3}{*}{0,03} \\
\hline percentil 90-95 & 21 & $(11,9)$ & 17 & $(13,0)$ & 38 & $(12,3)$ & \\
\hline percentil 95-99 & 79 & $(44,6)$ & 40 & $(30,5)$ & 119 & $(38,6)$ & \\
\hline \multicolumn{8}{|c|}{ Pressão arterial diastólica $(\mathrm{mm} / \mathrm{Hg})$} \\
\hline$<$ percentil 90 & 82 & $(46,3)$ & 66 & $(50,4)$ & 148 & $(48,1)$ & \multirow{3}{*}{0,77} \\
\hline percentil 90-95 & 30 & $(16,9)$ & 21 & $(16,0)$ & 51 & $(16,6)$ & \\
\hline percentil 95-99 & 65 & $(36,7)$ & 44 & $(33,6)$ & 109 & $(35,4)$ & \\
\hline
\end{tabular}

* Teste do Qui-quadrado.

A Tabela 2 apresenta a classificação do IMC, ApC, PAS e pressão arterial diastólica (PAD) de acordo com os pontos de corte e critérios de saúde (atende/não atende) adequados por idade e sexo. Nota-se que $12,3 \%$ dos adolescentes encontravam-se acima do peso; $75,3 \%$ apresentavam baixa ApC; e $51 \%$ apresentavam valores elevados de PAS e PAD. A prevalência de adolescentes que atendiam/não atendiam aos critérios de saúde para ApC e PAS foram estatisticamente diferentes entre meninos e meninas. A prevalência de baixa ApC foi maior nos meninos do que nas meninas. Em ralação à PAS, as meninas mais frequentemente tiveram níveis normais, enquanto os meninos mais frequentemente tiveram níveis aumentados.
A Tabela 3 apresenta as RC de não se atender critérios de saúde de PAS e PAD entre os adolescentes que não atendem os níveis de saúde para $\mathrm{ApC}$ e IMC. Quanto ao IMC, as associações brutas foram estatisticamente significativas, apontando que os adolescentes com excesso de peso apresentam maior $\mathrm{RC}$ de atingir níveis aumentados de PAS e de PAD do que os adolescentes que apresentaram normalidade para o IMC. Quando a análise foi realizada ajustada por idade e sexo, a força e o nível de significância da associação entre IMC e PAS e PAD aumentou. A ApC não apresentou associação bruta estatisticamente significativa com a PAS. Quando a análise foi realizada ajustada por idade e sexo, o nível de significância da associação entre ApC e PAS passou a ser limítrofe. 
Tabela 2. Classificação do índice de massa corporal, aptidão cardiorrespiratória, pressão arterial sistólica e pressão arterial diastólica de acordo com os pontos de corte e critérios de saúde (atende/ não atende) adequados por idade e sexo, em amostra de 361 estudantes com idade entre 14 e 17 anos, de uma escola pública em Fortaleza, Ceará

\begin{tabular}{|c|c|c|c|c|}
\hline Critérios de saúde & $\begin{array}{c}\text { Meninos } \\
\text { n (\%) }\end{array}$ & $\begin{array}{c}\text { Meninas } \\
\text { n (\%) }\end{array}$ & $\begin{array}{l}\text { Total } \\
\text { n (\%) }\end{array}$ & $\mathbf{p}^{*}$ \\
\hline \multicolumn{5}{|l|}{ Aptidão cardiorrespiratória } \\
\hline Atende (normal) & $30(16,9)$ & $46(35,1)$ & $76(24,7)$ & \multirow{2}{*}{0,01} \\
\hline Não atende (baixa) & $147(83,1)$ & $85(64,9)$ & $232(75,3)$ & \\
\hline \multicolumn{5}{|l|}{ Índice de massa corporal } \\
\hline Atende (normal) & $160(90,4)$ & $110(84,0)$ & $270(87,7)$ & \multirow{2}{*}{0,09} \\
\hline Não atende (alto) & $17(9,6)$ & $21(16,0)$ & $38(12,3)$ & \\
\hline \multicolumn{5}{|l|}{ Pressão arterial sistólica } \\
\hline Atende (normal) & $77(43,5)$ & $74(56,5)$ & $151(49,0)$ & \multirow{2}{*}{0,02} \\
\hline Não atende (alta) & $100(56,5)$ & $57(43,5)$ & $157(51,0)$ & \\
\hline \multicolumn{5}{|l|}{ Pressão arterial diastólica } \\
\hline Atende (normal) & $82(46,3)$ & $66(50,4)$ & $148(48,1)$ & \multirow{2}{*}{0,48} \\
\hline Não atende (alta) & $95(53,7)$ & $65(49,6)$ & $160(51,9)$ & \\
\hline
\end{tabular}

* Teste do qui-quadrado; diferença entre meninos e meninas.

Tabela 3. Associações entre pressão arterial sistólica e pressão arterial diastólica com índice de massa corporal e aptidão cardiorrespiratória em 361adolescentes com idade entre 14 e 17 anos, de uma escola pública em Fortaleza, Ceará

\begin{tabular}{|c|c|c|c|c|}
\hline Variáveis & RC bruta (IC 95\%) & $\mathbf{p}^{*}$ & RC ajustada (IC 95\%)† & p* \\
\hline \multicolumn{5}{|c|}{ Pressão arterial sistólica não atende (não normal) } \\
\hline \multicolumn{5}{|c|}{ Aptidão cardiorrespiratória } \\
\hline Atende (normal) & 1 & & 1 & \\
\hline Não atende (baixa) & $0,85(0,50-1,43)$ & 0,55 & $0,59(0,33-1,03)$ & 0,06 \\
\hline \multicolumn{5}{|c|}{ Índice de massa corporal } \\
\hline Atende (normal) & 1 & & 1 & \\
\hline Não atende (alto) & $3,02(2,94-6,13)$ & $<0,001$ & $3,12(3,80-6,83)$ & 0,01 \\
\hline \multicolumn{5}{|c|}{ Pressão arterial diastólica não atende (não normal) } \\
\hline \multicolumn{5}{|c|}{ Aptidão cardiorrespiratória } \\
\hline Atende (normal) & 1 & & 1 & \\
\hline Não atende (baixa) & $1,03(0,61-1,73)$ & 0,89 & $0,87(0,51-1,51)$ & 0,63 \\
\hline \multicolumn{5}{|c|}{ Índice de massa corporal } \\
\hline Atende (normal) & 1 & & 1 & \\
\hline Não atende (alto) & $3,41(1,55-7,49)$ & 0,02 & $3,70(1,66-8,24)$ & 0,01 \\
\hline
\end{tabular}

* Regressão logística binária.

+ Valores ajustados por sexo, idade e variáveis independentes inseridas no modelo de regressão.

$\mathrm{RC}$, razão de chances; IC, intervalo de confiança.

\section{DISCUSSÃO}

A transição entre a infância e a adolescência é um período de transformações bio-psico-sociais em que o estilo de vida e os padrões de comportamento alimentar e de atividade física estão sendo estruturados, os quais terão grandes implicações no risco de desenvolver alguma DCNT durante a própria infância e na vida adulta. ${ }^{4,12,21}$ Neste sentido, é muito relevante identificar a prevalência de fatores de risco em crianças e adolescentes, além de pesquisar as relações entre fatores tais como IMC, ApC e PA..$^{12,17}$

Este e outros estudos com adolescentes verificaram que os meninos apresentam geralmente valores médios 
estatisticamente maiores de peso, estatura e ApC, ${ }^{22}$ assim como de PAS e PAD do que as meninas. ${ }^{8,12,17}$ Sabe-se que até aproximadamente os 12 anos de idade estes valores são semelhantes para ambos os sexos, ocorrendo a diferenciação no final da adolescência, em função das mudanças biológicas que ocorrem com a puberdade..$^{8,23}$

A prevalência de fatores de risco na população estudada foi grande. Dados similares foram encontrados em outros estudos sobre IMC e ApC. ${ }^{3,6}$ Por outro lado, estudos realizados com adolescentes em diversas cidades brasileiras apontam valores discrepantes para a prevalência de HAS: 44,7\% em Fortaleza, Ceará, ${ }^{8}$ $17,3 \%$ em Recife, Pernambuco, ${ }^{13}$ e 5\% em Goiânia, Goiás. ${ }^{14}$ As diferentes metodologias adotadas para o diagnóstico da HAS em cada estudo podem justificar a variabilidade observada, dificultando a comparação dos resultados. Fatores como faixa etária estudada, número de visitas realizadas, número de aferições de PA em cada visita e intervalo entre as aferições contribuem de forma importante para a variação de prevalências. ${ }^{5}$ A elevada prevalência de HAS no presente estudo pode ser decorrente da mensuração da PA ter ocorrido em uma única visita. Araújo et al. ${ }^{8} \mathrm{e}$ Guedes et al. ${ }^{24}$ justificam que valores mais elevados de PA são encontradas em estudos que se baseiam em uma avaliação, e que os valores caem quando são realizadas duas ou três avaliações consecutivas.

Quando as prevalências dos fatores de risco foram analisadas separadas por sexo, observou-se uma tendência à maior prevalência de IMC elevado nas meninas, porém não foi estatisticamente significativa. Outros estudos reportam que as meninas tendem a ter aumento da massa de gordura maior que os meninos na adolescência. ${ }^{6,7}$ As diferenças identificadas nos valores de IMC entre os sexos podem ser justificadas de algumas maneiras: nível de maturação corporal, diferenças nos métodos de avaliação do estado nutricional e as próprias diferenças regionais, sugerindo a necessidade de novos estudos onde possam ser identificados os fatores relacionados a essas diferenças na composição corporal. . $^{2,25}$

A prevalência de baixa ApC foi maior entre os meninos do que entre as meninas, diferença esta também observada em outros estudos. ${ }^{3,12} \mathrm{O}$ desempenho no teste de ApC pode ser alterado pelo nível de motivação e espírito de competitividade do indivíduo. Apesar dessas características serem geralmente mais presentes nos meninos, pode acontecer que as meninas se dediquem mais ao realizar o teste, enquanto que os rapazes apresentam comportamento mais descontraído. Pode acontecer também que os protocolos não estejam utilizando pontos de corte adequados para identificar as diferenças de ApC entre os sexos..$^{2,12}$

A prevalência de PAS aumentada foi maior entre os meninos, não sendo a diferença estatisticamente significativa para PAD. Alguns estudos com adolescentes ${ }^{13,26}$ observaram prevalências maiores de HAS entre os indivíduos do sexo masculino, enquanto outros $^{27,28}$ encontraram maiores prevalências entre o sexo feminino. Há ainda outros estudos que não encontraram diferença na PA entre os sexos. ${ }^{5,14}$ Talvez a divergência de resultados entre os estudos possa estar associada a diferenças temporais em que ocorrem as alterações na composição corporal e produção de testosterona decorrentes da maturação sexual. ${ }^{8,15}$

Ao se analisarem as associações entre as variáveis estudadas, pode-se observar que o risco de apresentar PA elevada foi estatisticamente maior nos adolescentes com excesso de peso quando comparados aos que apresentaram IMC normal. Já com a $\mathrm{ApC}$ não houve associação significativa, mesmo considerando a regressão ajustada pelo sexo, IMC e ApC. Pinto et al. ${ }^{5}$ também apontaram essa relação entre IMC e PA. Uma pesquisa semelhante foi realizada com adolescentes americanos, verificando que aqueles que apresentavam excesso de peso também apresentavam valores elevados de PAS e PAD. ${ }^{25}$ Outra pesquisa realizada com adolescentes obesos nos Estados Unidos diagnosticou que $34,7 \%$ desses jovens estavam com pressão elevada: $27,9 \%$ destes apresentavam préhipertensão e $6,8 \%$, HAS. $^{29}$

A relação entre a ApC e a PA é menos evidente, e outros estudos também não identificaram associações significativas. ${ }^{16,22}$ Entretanto, alguns mostram que adolescentes com baixa ApC apresentaram maiores chances de atingir níveis indesejados de PA. ${ }^{12,17,30}$ Esta indefinição pode ser fortemente influenciada pelo efeito de variáveis de confusão, como o crescimento físico e as alterações hormonais, que se manifestam de maneira mais evidente durante esta fase do desenvolvimento humano. ${ }^{17,16}$

O presente estudo apresenta, como limitações, o desenho transversal que impossibilita o estabelecimento de relações de causa e efeito; a seleção amostral por conveniência, que não permite a extrapolação dos dados; a grande oscilação natural que ocorre nos valores de PA em indivíduos de idade pediátrica, principalmente quando a PA é aferida em uma única visita; ${ }^{31} \mathrm{e}$ o fato de não ter analisado o comportamento de outros parâmetros metabólicos importantes para a saúde. Mesmo considerando estas limitações, vale salientar a relevância dos resultados encontrados neste tipo de população. O estudo realizado em uma 
população em risco social com baixa renda econômica mostra que a prevalência dos fatores de risco antes atribuídos a populações mais ricas e emergentes afeta de forma preocupante também a população de nível socioeconômico desfavorecido. ${ }^{2,3}$ Estudos epidemiológicos têm demonstrado forte associação entre os fatores de risco encontrados e as DCV em jovens..$^{15,16}$

É importante ressaltar que foi encontrada alta prevalência de $\mathrm{ApC}$ inadequada. Levando em conta o conhecido fator protetor dos níveis adequados de ApC, que são inversamente associados à obesidade e HAS, ${ }^{8,15,16}$ o achado é preocupante. Estilos de vida mais saudáveis devem ser estimulados, em especial quanto à adoção dos hábitos alimentares e de prática regular da atividade física durante a infância e adolescência. ${ }^{2}$

Os dados sugerem que adolescentes de classe econômica baixa apresentam alto risco de desenvolver DCV precocemente. O excesso de peso, a baixa ApC e a PA acima do normal foram prevalentes nesta população. Como a interação entre estes fatores de risco pode potencializar os problemas de saúde, e tendo em vista as graves conseqüências associadas às DCV e DCNT em geral, recomenda-se que medidas de vigilância, prevenção e promoção à saúde sejam realizadas nas escolas, a fim de modificar a realidade apresentada.

\section{AGRADECIMENTOS}

À direção e aos professores do Colégio Liceu, Fortaleza, CE, pelo apoio à pesquisa.

\section{REFERÊNCIAS}

1. World Health Organization. The Global Burden of Disease - 2004 Update. [Internet]. Geneva; 2008. [cited 2012 Aug]. Available at: http:// www.who.int/healthinfo/global_burden_disease/GBD_report_2004update_full.pdf?ua=1

2. Souza EA, Barbosa Filho VC, Nogueira JAD, Azevedo Júnior MR. Atividade física e alimentação saudável em escolares brasileiros: revisão de programas de intervenção. Cad Saúde Pública. 2011;27(8):1459-71.

3. Petroski EL, Silva AF, Rodrigues AB, Pelegrini A. Aptidão física relacionada a saúde em adolescentes brasileiros residentes em áreas de médio/baixo índice de desenvolvimento humano. Rev Salud Pública. 2011;13(2):219-28.

4. Juonala M, Järvisalo MJ, Mäki-Torkko N, Kähönen M, Viikari JS, Raitakari OT. Risk factors identified in childhood and decreased carotid artery elasticity in adulthood: the Cardiovascular Risk in Young Finns Study. Circulation. 2005;112(10):1486-93.

5. Pinto SL, Silva RC, Priore SE, Assis AMO, Pinto EJ. Prevalência de pré-hipertensão e de hipertensão arterial e avaliação de fatores associados em crianças e adolescentes de escolas públicas de Salvador, Bahia, Brasil. Cad Saúde Pública. 2011;27(6):1065-76.

6. Bergmann GG, Gaya A, Halpern R, Bergmann MLA, Rech RR, Constanzi CB, Alli LR. Índice de massa corporal para triagem de fatores de risco para doenças cardiovasculares na infância. Arq Bras Endocrinol Metab. 2011;55(2):114-20.

7. Barbosa Filho VC, Reges LAG, SOUZA EA, Ribeiro EAG, Lima AB. Práticas esportivas e recreativas em adolescentes com excesso de peso: análise da composição corporal e do desempenho motor. Motriz. 2011;17(2):264-73.

8. Araújo TL, Lopes MVO, Cavalcante TF, Guedes NG, Moreira RP, Chaves ES, Silva VM. Análise de indicadores de risco para hipertensão arterial em crianças e adolescentes. Rev Esc Enferm USP. 2008;42(1):120-26.

9. Marchi-alves LM, Mazzo A. Obesidade infantil ontem e hoje: importância da avaliação antropométrica pelo enfermeiro. Esc Anna Nery 2011;87(1):238-44.

10. Brasil. Ministério da Saúde. Vigitel Brasil 2010: Vigilância de fatores de risco e proteção para doenças crônicas por inquérito telefônico. [Internet]. Brasília (DF); 2011. [cited 2012 Aug]. Available at: http://portal.saude.gov.br/portal/arquivos/pdf/vigitel2010_final_web.pdf

11. Brasil. Ministério do Planejamento, Orçamento e Gestão e Ministério da Saúde. POF Brasil: Pesquisa de Orçamentos Familiares. Instituto Brasileiro de Geografia e Estatística - IBGE: Rio de Janeiro; 2010.

12. Burgos MS, Reuter CP, Burgos LT, Pohl HH, Pauli LTS, Horta JA, Reckziegel MB, Franke SIR, Prá D. Uma análise entre índices pressóricos, obesidade e capacidade cardiorrespiratória em escolares. Arqu Bras Cardiol. 2010;94(6):788-93.

13. Gomes BMR, Alvez JGBA. Prevalência de hipertensão arterial e fatores associados em estudantes de Ensino Médio de escolas públicas da Região Metropolitana do Recife, Pernambuco, Brasil, 2006. Cad Saúde Pública. 2009;25(2):375-81.

14. Monego ET, Jardim PCBV. Determinantes de risco para doenças cardiovasculares em escolares. Arqu Bras Cardiol. 2006;87(1):37-45.

15. Anderssen SA1, Cooper AR, Riddoch C, Sardinha LB, Harro M, Brage S, Andersen LB. Low cardiorespiratory fitness is a strong predictor for clustering of cardiovascular disease risk factors in children independent of country, age and sex. Eur J Cardiovasc Prev Rehabil. 2007; 14(4):526-31.

16. Carnethon MR, Gulati M, Greenland P. Prevalence and cardiovascular disease correlates of low cardiorespiratory fitness in adolescents and adults. JAMA. 2005;294(23):2981-8.

17. Fernandes RA, Casonatto J, Christofaro DGD, Cucato GG, Romanzini M, Ronque ERV. Excesso de peso e pressão arterial elevada em adolescentes blood pressure in adolescents. Rev Bras Med Esporte 2010;16(6):404-07.

18. PROESP-BR. Projeto Esporte Brasil: Manual. [Internet]. Porto Alegre; 2009. [cited 2010 May 10]. Available at: http://www.proesp.ufrgs.br. 
19. World Health Organization. Growth reference data for 5-19 years, WHO reference 2007. [Internet]. [cited 2014 Aug 4]. Available at: http://www.who.int/growthref/en/

20. National High Blood Pressure Education. Program Working Group on High Blood Pressure in Children and Adolescents: the fourth report on the diagnosis, evaluation, and treatment of high blood pressure in children and adolescents. Pediatrics. 2004;114:555-76.

21. Brito AKA, Silva Júnior FL, Coelho LS, França NM. Nível de atividade física e correlação com o índice de massa corporal e percentual de gordura em adolescentes escolares da cidade de Teresina-PI. Rev Bras Ativ Fís Saúde. 2012;17(3):212-16.

22. Pereira CH, Ferreira DS, Copetti GL, Guimarães LC, Barbacena MM, Liggeri N, Castro OG, Lobato S, de David AC. Aptidão física em escolares de uma unidade de ensino da rede pública de Brasília-DF. Rev Bras Ativ Fís Saúde. 2011;16(3):223-7.

23. de Visser DC, van Hooft IM, van Doornen LJ, Hofman A, Orlebeke JF, Grobbee DE. Anthropometric measures, fitness and habitua physical activity in off-spring of hypertensive parents. Dutch Hypertension and Offspring Study. Am J Hypertens. 1994; 7(3):242-48.

24. Guedes DP, Guedes JERP, Barbosa DS, Oliveira JA, Stanganelli LCR. Fatores de Risco Cardiovasculares am adolescentes: Indicadores Biológicos e Comportamentais. Arqu Bras Cardiol. 2006;86(6):439-50.

25. Falkner B, Gidding SS, Ramirez-Garnica G, Wiltrout SA, West D, Rappaport EB. The relationship of body mass index and blood pressure in primary care pediatric patients. J Pediatr. 2006; 148(2):195-200.

26. Nur N, Cetinkaya S, Yilmaz A, Ayvaz A, Bulut MO, Sümer H. Prevalence of hypertension among high school students in a middle Anatolian province of Turkey. J Health Popul Nutr. 2008; 26:88-94.

27. Borges LMP, Peres MA, Horta BL. Prevalência de níveis pressóricos elevados em escolares de Cuiabá, Mato Grosso. Rev Saúde Pública. 2007;41(4):530-38.

28. Ferreira JS, Aydos RD. Prevalência de hipertensão arterial em crianças e adolescentes obesos. Ciênc Cuid Saúde. $2010 ; 15(1): 97-104$.

29. Boyd GS, Koenigsberg J, Falkner B, Gidding S, Hassink S. Effect of obesity and high blood pressure on plasma lipid levels in children and adolescents. Pediatrics. 2005;116(2):442-6.

30. Klasson-Heggebø L, Andersen LB, Wennlöf AH, Sardinha LB, Harro M, Froberg K, Anderssen SA. Graded associations between cardiorespiratory fitness, fatness, and blood pressure in children and adolescents. Br J Sports Med. 2006;40(1):25-9.

31. National high Blood Pressure Education Program Working Group High Blood Pressure in Children and Adolescents. The Fourth Report on the Diagnosis, Evaluation, and Treatment of High Blood Pressure in Children and Adolescents. Pediatrics. 2004;114(2):555-76. 